\title{
Indices of socio-economic development in the space of characteristics of regional differentiation
}

\author{
Mikhail Y. Afanasiev", Aleksander V. Kudrov, and Maria A. Lysenkova \\ Central Economics and Mathematics Institute of the Russian Academy of Sciences, Moscow, Russia
}

\begin{abstract}
As a rule, the source information and methods used for construction of many indices have specifics that allow authors and experts to consider these indexes as original and recommend them for use in solving a number of management tasks. However, a comparative analysis of such indices sometimes shows that despite the peculiar names and methods of calculation, some of them do not have significant statistical differences. This paper presents an approach that allows us to compare an arbitrary set of indices and ratings that characterize the level of socioeconomic development of the subjects of the Russian Federation in the space of characteristics of differentiation used in solving regional management problems. The purpose of the study is to identify indexes that do not differ significantly. Eighteen indexes are compared. At the first stage of the study, their correlation analysis is performed. Then the indices are regressed to the basic characteristics of differentiation. The analysis of cosines of angles between indices calculated using beta coefficients of regressions allows us to refine the results of correlation analysis and identify a subset of indices that are not distinguishable in solving regional management problems.
\end{abstract}

\section{Introduction}

Many indices have been published that characterize various socio-economic aspects of regional development (Batchaev,2000). The source information and methods used for their construction have specifics that allow authors and experts to consider these indexes as original and recommend them for use in solving a number of management tasks. However, a comparative analysis of such indices sometimes shows that despite the peculiar names and methods of calculation, some of them do not have significant statistical differences. This paper presents an approach that allows us to compare an arbitrary set of indices and ratings that characterize the level of socio-economic development of the subjects of the Russian Federation in the space of characteristics of differentiation used in solving regional management problems. Here we proceed from the fact that the solution of a specific problem of regional management is based on the application of an economic and mathematical model parameterized using a set of characteristics of regional differentiation.

\footnotetext{
*Corresponding author: mi.afan@yandexi.ru
} 
The use of characteristics of differentiation is a natural direction of development of models for solving regional management problems in the digital economy. The issues related to the formation of a space of such characteristics in the context of solving problems of project management are considered in (Aivazian, Afanasiev, \&Kudrov, 2019). It will be shown that indexes and ratings that have a significant difference in the author's representation may be indistinguishable in the space of characteristics of regional differentiation.

The comparison of eighteen indices of socio-economic development of Russian regions is carried out. Some of them are constructed in the space of characteristics of differentiation calculated using theoretically based models of regional development. To construct these indices, the basis $\left.B_{t}^{\square}=\left\{l_{k, t}, s_{k, t}^{1}, s_{k, t}^{2}, t e_{k, t}, d t e_{k, t}\right)\right\}_{k}$ of characteristics of regional differentiation was used, which at a given time $t$ includes five components. The Rosstat indicator "number of economically active population" is used as a characteristic $l_{k, t}$ of the scale of the economy. The basis includes the first $s_{k, t}^{1}$ (index of industry specialization) and the second $s_{k, t}^{2}$ (index of industrialization) principal components of the GRP structure. Based on the concept of a stochastic frontier (Kumbhakar,\&Lovell, 2004), using production functions that determine the dependence of GRP on the volume of main production factors, estimates of the technical efficiency of regions are obtained. The basis also includes estimates $d t e_{k, t}$ of the trend of technical efficiency, $d t e_{k, t}=t e_{k, t}-t e_{k, t-1}$. The paper (Aivazianet al.,2019) presents the conditions that the characteristics of differentiation must meet, so that they can be used as an basis for building indices of the main directions of socio-economic development: lack of high correlation; they should differentiate well the factors that determine the specifics of the indexes; to influence the index of at least one direction of socio-economic development; to have an economic interpretation. It was shown that the basis $\left.B_{t}^{\square}=\left\{l_{k, t}, s_{k, t}^{1}, s_{k, t}^{2}, t e_{k, t}, d t e_{k, t}\right)\right\}_{k}$ components satisfy these conditions.

The basis used in this work has been tested in the development of a methodology for assessing the quality of life, taking into account the characteristics of regional differentiation (Afanasiev, \&Kudrov, 2019).However, other sets of characteristics of differentiation can be used to assess the indistinguishability of socio-economic development indices. They can be formed depending on the tasks of regional management.

This paper uses the characteristics of differentiation estimated according to 2016 data. The following 7 indices are constructed in the space of characteristics of differentiation using indicators of socio-economic development for 2016 . $\mathrm{IB}^{1}$ — production of goods and services, volumes"; $\mathrm{IB}^{2}$ — "material welfare"; $\mathrm{IB}^{3}$ - "production of goods and services per capita"; IB ${ }^{4}$ —"quality of the social sphere"; IB $^{5}$ — "social security"; IB $^{6}$ — "demography"; $\mathrm{IB}^{7}$ - "health". A more detailed description of the analytical solution to this problem and the coordinates of $\mathrm{IB}^{1}, \ldots, \mathrm{IB}^{7}$ indices in the space of characteristics of differentiation are presented in (Aivazianet al.,2019).

The AM index of the material basis of quality of life is constructed as the first principal component of five indices $\mathrm{IB}^{1}, \ldots, \mathrm{IB}^{5}$. The AQL quality of life index is constructed as a linear combination of the $\mathrm{AM}$ index, $\mathrm{IB}^{6}$ and $\mathrm{IB}^{7}$ indices (Afanasiev, \&Kudrov, 2019). The AEC economic complexity index is based on a generalization of the approach to assessing economic complexity described in (Hidalgo,\&Hausmann, 2009). The procedure and data for constructing the AEC economic complexity index is described in (Afanasyev, \&Kudrov, 2020). The ER index of economic complexity is based on data 2016. The index methodology is based on the traditional approach to assessing economic complexity on product exports data by Russian regions, taking into account data on product exports by national economies of other countries (Lyubimov,Gvozdeva,\&Kazakova,2017). Quality of 
life index (rating) QL1 is based on data 2017. The 72 indicators are grouped into 11 groups that characterize the main aspects of quality of life in the region (RIA Rating, 2017).Index of socio-economic status of the subjects of the Russian Federation QL2 is based on data 2017. The integral rating was defined as the geometric average of rating points of 4 groups of indicators. The rating score for each group was determined as the arithmetic average of the rating points of all indicators included in the group: indicators of economic scale, indicators of economic efficiency, indicators of the budget sector, and indicators of the social sphere (RIA Rating,2018). The CA index of competitiveness of Russian regionsis based on data 2015. The index takes into account indicators that reflect the results of interregional competition: markets, institutions, human capital, innovation, natural resources, investment and financial capital (Leontief Centre ..., 2015). The investment attractiveness POT rating of Russian regions is based on data 2016. Investment attractiveness is assessed by two parameters: investment potential and investment risk. The potential shows what share the region occupies in the all-Russian market (labor, financial, production, consumer, institutional, infrastructure, natural resources, tourism and innovation), and the risk - what the scale of certain problems in the region may be for the investor (financial, social, managerial, economic, environmental, and criminal) (RAEX Rating, 2017). The index of credit status of regions FIN is based on data 2017. The index methodology is based on the analysis of indicators that characterize the ability of a subject of the Russian Federation to meet its debt obligations. All analyzed indicators are grouped into three groups: debt burden; budget and economy. The integral estimate of a region is defined as the geometric mean of the estimates of all groups of indicators. The estimation of a group is defined as the geometric average of all the included indicators (RIA Rating Review, 2017).

All characteristics of differentiation, as well as indexes $\mathrm{IB}^{1}, \ldots, \mathrm{IB}^{7}, \mathrm{AM}, \mathrm{AQL}, \mathrm{AEC}$ are estimated for 80 regions. The names of regions are specified, for example, in (Aivazianet al.,2019).For this set of regions, all the indices considered in this work are compared. All analyzed indices and characteristics of differentiation are normalized with an average of 0 and a standard deviation of 1 . To correctly compare indexes using regional characteristics of differentiation, the following conditions should be taken into account: 1) indexes and characteristics of differentiation should be built on the basis of comparable data over time; 2) the characteristics of differentiation should not have a strong correlation that cause a multicollinearity effect when used as regressors.

\section{Methods}

A correlation analysis of indexes is performed at the first stage of the study. At this stage, the influence of differentiation characteristics is not taken into account. Accordingly, the problem of identifying indexes that are indistinguishable in the space of characteristics of regional differentiation is not considered. If the modules of the index correlation coefficients with all other indexes are close to zero, then we exclude this index from consideration as having a significant difference from the others and independent. Correlation analysis allows you to reduce the number of indicators analyzed in the following stages.

At the second stage, projections of the indices are constructed in the space of characteristics of differentiation. Some indexes (for example, IB1,.., IB7) were initially built in this space. For the other indexes, their regressions on the characteristics of differentiation are built. Based on the analysis of beta coefficients, the characteristics of differentiation may by identified that have a significant impact on the indexes under consideration. This allows you to refine the economic interpretation of indexes. 
At the third stage, the cosines of the angles for each pair of indices in the space of characteristics of differentiation are calculated. Beta coefficients are coordinates of indices in the space of characteristics of differentiation and are used for cosines calculating. The calculated cosine value is compared with the threshold value, which is set by experts. If the cosine value exceeds the threshold value, the indexes are recognized as indistinguishable in the space of characteristics of differentiation. In this study, we used two threshold values of the cosines: 0.966 which corresponds to the angle $15^{\circ}$ and 0.985 which corresponds to the angle $10^{\circ}$. Other thresholds can also be used.

The use of cosines to identify indistinguishable indices is possible in the space of characteristics of differentiation only. Comparison of indices can also be carried out on the basis of correlation analysis of their projections into the space of characteristics of differentiation. But the use of cosines makes it easier to fix the indistinguishability of indexes, since their relationship with the angle between vectors in the space of characteristics of differentiation is traced.

\section{Results}

Table 1 shows the Pearson correlation coefficients of all the analyzed indices, with the exception of the $\mathrm{IB}^{1}, \ldots, \mathrm{IB}^{7}$ indices of the main directions of socio-economic development. Indices $\mathrm{IB}^{1}, \ldots, \mathrm{IB}^{7}$ determined by the values of characteristics of differentiation are not considered at this stage, since their coordinates in the space of characteristics of differentiation are known by construction. The correlation coefficients in italics exceed the value 0.8 . We can assume that the indices QL1, QL2, CA, AT, FIN characterize the material basis of the quality of life and have a close application orientation. We can also assume that the AEC and, ER indices of economic complexity are specific to the indices that characterize the material basis of quality of life.

Table 1. Correlation matrix of 9indices

\begin{tabular}{|l|c|c|c|c|c|c|c|c|}
\hline & AM & AQL & AEC & QL1 & QL2 & ER & CA & POT \\
\hline AQL & $\mathbf{0 . 8 5}$ & 1 & & & & & & \\
\hline AEC & -0.10 & 0.15 & 1 & & & & & \\
\hline QL1 & 0.78 & 0.66 & 0.05 & 1 & & & & \\
\hline QL2 & 0.79 & 0.53 & -0.22 & $\mathbf{0 . 8 2}$ & 1 & & & \\
\hline ER & 0.51 & 0.46 & 0.18 & 0.54 & 0.46 & 1 & & \\
\hline CA & 0.79 & 0.57 & -0.23 & 0.78 & $\mathbf{0 . 8 9}$ & 0.47 & 1 & \\
\hline POT & 0.64 & 0.45 & -0.13 & 0.72 & $\mathbf{0 . 8 8}$ & 0.46 & $\mathbf{0 . 8 6}$ & 1 \\
\hline FIN & 0.65 & 0.42 & -0.27 & 0.68 & $\mathbf{0 . 8 3}$ & 0.35 & 0.79 & 0.78 \\
\hline
\end{tabular}

Table 2 shows the coefficients of determination and estimates of beta regressions coefficients for all the indices under consideration on the five above-mentioned characteristics of differentiation. A separate line for each index contains the p-values of beta coefficients estimates. In table 4 , the indexes are presented in lexicographic order by the significance of beta coefficients estimates. 
Table 2. Beta coefficients of indexes regression on characteristics of differentiation

\begin{tabular}{|c|c|c|c|c|c|c|}
\hline & $\mathrm{R}^{2}$ & 1 & $\mathrm{Te}$ & s1 & s2 & dte 1 \\
\hline \multirow[t]{2}{*}{ AQL } & 0.784 & 0.759 & 0.276 & -0.187 & -0.258 & -0.169 \\
\hline & $\mathrm{P}>\mathrm{t}$ & 0 & 0 & 0.001 & 0 & 0.003 \\
\hline \multirow[t]{2}{*}{ ER } & 0.513 & 0.523 & -0.007 & -0.291 & 0.249 & -0.009 \\
\hline & $\mathrm{P}>\mathrm{t}$ & 0 & 0.93 & 0.001 & 0.004 & 0.907 \\
\hline \multirow[t]{2}{*}{$\mathrm{AM}$} & 0.829 & 0.807 & 0.218 & 0.092 & 0.127 & 0.065 \\
\hline & $P>t$ & 0 & 0 & 0.068 & 0.013 & 0.179 \\
\hline \multirow[t]{2}{*}{ QL1 } & 0.603 & 0.573 & 0.151 & -0.176 & 0.281 & 0.115 \\
\hline & $\mathrm{P}>\mathrm{t}$ & 0 & 0.057 & 0.023 & 0 & 0.121 \\
\hline \multirow[t]{2}{*}{ QL2 } & 0.751 & 0.660 & 0.009 & 0.149 & 0.442 & 0.058 \\
\hline & $\mathrm{P}>\mathrm{t}$ & 0 & 0.875 & 0.016 & 0 & 0.315 \\
\hline \multirow[t]{2}{*}{$\mathrm{AEC}$} & 0.653 & -0.131 & 0.094 & -0.797 & -0.207 & 0.133 \\
\hline & $\mathrm{P}>\mathrm{t}$ & 0.071 & 0.204 & 0 & 0.005 & 0.056 \\
\hline \multirow[t]{2}{*}{$\mathrm{CA}$} & 0.751 & 0.724 & 0.076 & 0.101 & 0.316 & 0.083 \\
\hline & $\mathrm{P}>\mathrm{t}$ & 0 & 0.224 & 0.097 & 0 & 0.157 \\
\hline \multirow[t]{2}{*}{ POT } & 0.641 & 0.655 & 0.014 & -0.010 & 0.333 & 0.084 \\
\hline & $\mathrm{P}>\mathrm{t}$ & 0 & 0.844 & 0.886 & 0 & 0.232 \\
\hline \multirow[t]{2}{*}{ FIN } & 0.559 & 0.536 & 0.077 & 0.071 & 0.383 & 0.124 \\
\hline & $\mathrm{P}>\mathrm{t}$ & 0 & 0.391 & 0.375 & 0 & 0.113 \\
\hline
\end{tabular}

Table 3 shows the cosines of angles between indices that characterize the quality of life, financial situation of regions, and their economic complexity. As a threshold value for determining are the indices indistinguishable or not we uses the expert value of the cosine of the angle $15^{\circ}$ equal to 0.966 . The cosines of the angles between the six index pairs (QL2, CA), (QL2, POT), (QL2, FIN), (CA, POT), (CA, FIN), (POT, FIN) exceed the threshold value of 0.966 . This means that in the space of characteristics of differentiation the angles between any pair of indices from the subset QL2, CA, POT, FIN are less than $15^{\circ}$. We can conclude that the indices QL2, CA, POT, FIN are indistinguishable in the space of characteristics of differentiation. At the same time, the difference between any other index pairs is significant.

Table 3. The cosines of the angles between indices

\begin{tabular}{|c|c|c|c|c|c|c|c|c|}
\hline & AM & AQL & AEC & QL1 & QL2 & ER & CA & POT \\
\hline AQL & 0.81 & 1 & & & & & & \\
\hline AEC & -0.24 & 0.14 & 1 & & & & & \\
\hline QL1 & 0.88 & 0.68 & 0.06 & 1 & & & & \\
\hline QL2 & 0.88 & 0.49 & -0.41 & 0.86 & 1 & & & \\
\hline ER & 0.76 & 0.67 & 0.2 & 0.93 & 0.78 & 1 & & \\
\hline CA & 0.95 & 0.64 & -0.32 & 0.91 & 0.979 & 0.81 & 1 & \\
\hline POT & 0.91 & 0.61 & -0.21 & 0.94 & 0.972 & 0.89 & 0.986 & 1 \\
\hline FIN & 0.88 & 0.49 & -0.31 & 0.91 & 0.986 & 0.80 & 0.976 & 0.976 \\
\hline
\end{tabular}


With a threshold value of 0.985 corresponding to the angle of $10^{\circ}$, only two pairs of indexes (QL2, FIN) and(CA, POT) are indistinguishable. At the threshold value of 0.987 , corresponding to the angle of $9^{0}$, there are no indistinguishable indexes. At the same time, the difference between any other index pairs is significant. With a threshold value of 0.985 corresponding to the angle of $10^{\circ}$, only two pairs of indexes (QL2, FIN) and(CA, POT) are indistinguishable. At the threshold value of 0.987 , corresponding to the angle of $9^{0}$, there are no indistinguishable indexes.

The maximum value 0.957 of the cosine of the angle between indices $\mathrm{IB}^{1}, \ldots, \mathrm{IB}^{7}$ and other indices in the space of characteristics of differentiation. The maximum does not reach the 0.966 threshold. It can be concluded that the indices $\mathrm{IB}^{1}, \ldots, \mathrm{IB}^{7}$ differ significantly in the space of characteristics of differentiation from other indices.

\section{Discussion}

It is not difficult to see how close are estimates of regions based on projections of indexes QL2, CA, POT, FIN are indistinguishable in the space of characteristics of differentiation. To do this, we construct projections of these indices in the space of characteristics of differentiation using the beta regression coefficients shown in table 4.The Spearman rank correlation coefficient value for any pair of indexes from the set QL2, CA, POT, FIN is greater than 0.96 . With such high rank correlation coefficients, we can expect that only a small number of regions will have a significant deviation in the ranks for the indexes under consideration. Indeed, in figure 1, the ranks difference for the QL2 and FIN index projections that exceeds modulo 8 (10\% of the maximum possible difference) is observed for only three regions. For other pairs of indexes, the difference in rank exceeding modulo 8 is observed for no more than 4 regions. Thus, using any of the four indistinguishable indexes results in close rank estimates for $95 \%$ of the regions.

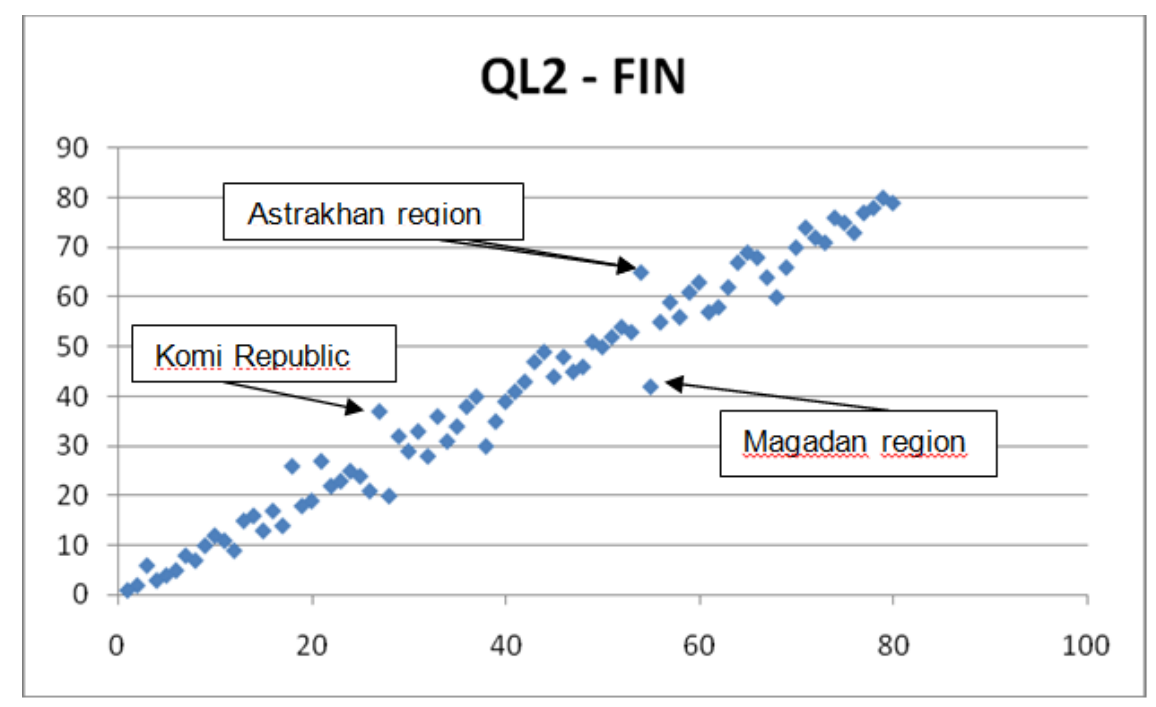

Fig. 1.The relationshipof QL2 and FIN indices projections. On the abscissus axis, there are ranks of regions according to the QL2 index. On the ordinate axis - according to the FIN index.

It is not difficult to compare the regions that have the best and worst values for indexes in the author's representation with regions that have the best and worst values of these indices projections in the space of characteristics of differentiation. The top eleven for each index in the author's representation includes only five regions: Moscow, St. Petersburg, 
Moscow region, Republic of Tatarstan, Republic of Bashkortostan. Only three regions are among the ten worst in each index: Jewish Autonomous region, Republic of Kalmykia, Karachay-Cherkess Republic. It can be noted that the indexes have a significant originality in the author's view. Sets of the eleven regions with the best values of indexes projections match for all four indexes. Sets of ten regions that have the worst values of indexes projections also match for all four indexes. This is another interpretation of the indistinguishability of the QL2, CA, POT and FIN indices projections in the space of characteristics of differentiation.

\section{Conclusion}

Correlation analysis of AM, QL1, QL2, CA, POT, FIN indices, that characterize the socioeconomic development of Russian regions, shows that they has a close applied orientation and characterize the material basis of the quality of life. At the same time, the AEC, ECIR indices of economic complexity are specific against the indices that characterize the material basis of life.

The cosines of the angles between the QL2, CA, POT, FIN indexes exceed the threshold value of 0.966 , corresponding to the angle of $15^{\circ}$. Therefore, we can conclude that these indices are indistinguishable in the space of characteristics of differentiation. At the same time, the difference between any other index pairs is significant. The use of cosines makes it easier to fix the indistinguishability of indexes, since their relationship with the angle between vectors in the space of characteristics of differentiation is traced.

The conclusion that the QL2, CA, POT and FIN indexes are indistinguishable should not be considered as a negative characteristic of them. In the case of indistinguishability, it does not matter which of these indexes to use in solving management problems. At the same time, an attempt to denote the originality of indistinguishable indexes based on differences in names or construction methods leads to a distortion of the perception of the management model parameterized using the characteristics of differentiation.

\section{Acknowledgment}

The work was supported by the Russian Foundation for Basic Research (RFBR project 20010-00223)

\section{References}

1. S.A. Aivazian, M.Yu. Afanasiev, A.V. Kudrov, Applied Econometrics, 54, 51 (2019)

2. M.Yu. Afanasiev, A.V. Kudrov, Journal of Economics, 15(3),7 (2019)

3. M.Yu. Afanasiev, A.V. Kudrov, Montenegrin Journal of Economics, 16 (4), 61 (2020).

4. A.R. Batchaev, Ratings. ICSER "Leontief centre", StratPlan.ru (2000), https://stratplan.ru/

5. C.A. Hidalgo, R. Hausmann, Proceedings of the National Academy of Sciences, 106 (26), 10570 (2009)

6. S. Kumbhakar, K. Lovell, Cambridge University Press (2004)

7. Leontief Centre - AV Group, The index of competitiveness of regions (2015), http://av-group.ru/

8. I. L. Lyubimov, M. A. Gvozdeva, M. V. Kazakova, Journal NEA, 2 (34), 94 (2017) 
9. RAEX Rating, Regional investment climate (2017), .https://raex-a.ru/

10. RIA Rating. Rating of the socio-economic situation of the regions (2018), https://riarating.ru/

11. RIA Rating, Review The index of creditworthiness of the regions (2017), http://vid1.rian.ru/ 